\title{
Using Optical Coherence Tomography (OCT) to Evaluate Human Donor Kidneys Prior to and Following Transplantation
}

\section{Peter M Andrews ${ }^{1 *}$ and Yu Chen ${ }^{2}$}

${ }^{1}$ Georgetown University Medical Center, Washington, DC, USA

${ }^{2}$ Fischell Department of Bioengineering, University of Maryland, College Park, MD, USA

\begin{abstract}
The most common insult to donor kidneys destined for transplantation is Acute Tubular Necrosis (ATN). The extent of ATN will affect post-transplant function and is a significant risk factor for long-term graft function and survival. Optical Coherence Tomography (OCT) is a rapidly emerging imaging modality that can function as a type of "optical biopsy", providing non-invasive images of tissue morphology in situ and in real-time. In this paper, we review studies that support the use of OCT and Doppler based OCT (i.e., DOCT) to image the renal microstructure and blood flow of human donor kidneys. We conclude that OCT/DOCT imaging of donor kidneys prior to and following transplantation can provide transplant surgeons with a means for predicting ATN and post-transplant renal function.
\end{abstract}

Keywords: Optical Coherence Tomography (OCT); Doppler Optical Coherence Tomography (DOCT); Acute Tubular Necrosis (ATN); Delayed Graft Function (DGF); End-Stage Renal Disease (ESRD); Kidney transplantation; Uriniferous tubules; Renal blood flow

\section{Imaging Donor Kidneys to Determine their Status}

End-stage Renal Disease (ESRD) is associated with both high mortality rates and an enormous economic burden [1]. The preferred treatment option for ESRD that can extend patients' lives and improves their quality of life is kidney transplantation. However, ischemic insult suffered by kidneys awaiting transplantation frequently causes acute tubular necrosis (ATN) that leads to varying degrees of delayed graft function (DGF) after transplantation. Also, ATN represents a significant risk for eventual graft and patient survival $[2,3]$, and can be difficult to discern from rejection. In present clinical practice, there is no reliable real-time test to determine the viability of donor kidneys and whether or not donor kidneys might exhibit ATN. Therefore, there is a critical need for objective and reliable real-time tests to predict ATN to use these organs safely and utilize the donor pool optimally.

Previously it has been shown that the non-invasive imaging techniques (i.e., tandem scanning confocal microscopy-TSCM) could be used to determine the degree of ATN by analyzing the superficial nephrons of living kidneys in animal models and that these observations correlate with post-transplant renal function $[4,5]$. This is not surprising in that the status of superficial proximal convoluted tubules is indicative of the status of proximal convoluted throughout the entire kidney cortex. Non-invasive microscopic techniques are necessary for this determination because conventional microscopy results in artifacts that are difficult to distinguish from ATN [6]. Other investigators have also used near-infrared confocal microscopy [7] and multi-photon microscopy (MPM) [8-10] to demonstrate the ability to perform non-invasive imaging of kidney structure and function in animal models. However, the maximum penetration depth of those techniques for kidney imaging is very limited $(\approx 100 \mu \mathrm{m}$ for TSCM, and $\approx 200-300 \mu \mathrm{m}$ for MPM), which makes it difficult to impossible to nondestructively image the human kidney, especially if an intact human renal capsule surrounds it. Indeed, in a previous clinical trial, we found that the limited penetrating ability of TSCM precluded us from imaging human donor kidneys even when an attempt was made to remove the renal capsule [unpublished observations]. Also, conventional bulky systems like TSCM and MPM are awkward and especially difficult when attempting to image the kidney in the clinical arena. Therefore, a non-invasive microscopic procedure that has enough penetrating ability to image the human kidney parenchyma and determine the extent of ATN would provide invaluable clinical information regarding kidney function.

OCT is a rapidly emerging imaging modality that can function as a type of "optical biopsy", providing cross-sectional images of tissue morphology in situ and in real-time [11,12]. OCT is similar to ultrasound imaging, except that it uses the echo delay of light instead of sound to generate images. OCT is safer than X-ray technologies, much less expensive than MRI devices, and provides higher resolution images than ultrasound. By employing broadband optical light sources, OCT can achieve axial resolutions of 1-10 $\mu \mathrm{m}$, more than an order of magnitude above that obtainable for clinical ultrasound. As a result, OCT can provide very high- resolution images of organs and tissues in a non-invasive manner. This potential has been demonstrated in a number of biomedical applications including ophthalmology [1315], cardiology [16,17], gastroenterology [18-21], dermatology [22], dentistry [23], urology [24] and gynecology [25], among others. In contrast to other forms of non-invasive light microscopy, OCT can image with longer working distances, improved penetration depth and without the need for tissue contact. Not only can it image up to depths of $\sim 1-2 \mathrm{~mm}$ in most light- scattering tissues, OCT can also provide three-dimensional images in arbitrary planes. Finally, OCT can be performed using a thin flexible sterile endoscope or catheter [26,27] or even with a needle [28], enabling ease of use for minimallyinvasive procedures and the possibility of imaging deep within a solid tissue or organ. Previously, we have demonstrated that OCT is able to provide clinically relevant information (morphology and blood flow) using a rat kidney model [29-31]. In addition, we showed that OCT has sufficient penetration depth and resolution to visualize human

*Corresponding author: Peter M Andrews, Georgetown University Medical Center Washington, DC, USA, Tel: 202-687-1228; E-mail: andrewsp@georgetown.edu

Received December 31, 2013; Accepted January 23, 2014; Published January 27, 2014

Citation: Andrews PM, Chen Y (2014) Using Optical Coherence Tomography (OCT) to Evaluate Human Donor Kidneys Prior to and Following Transplantation. J Nephrol Ther 4: 151. doi:10.4172/2161-0959.1000151

Copyright: (c) 2014 Andrews PM, et al. This is an open-access article distributed under the terms of the Creative Commons Attribution License, which permits unrestricted use, distribution, and reproduction in any medium, provided the original author and source are credited. 
kidney microanatomy on intact human kidneys ex vivo even when surrounded by an intact connective tissue renal capsule [32,33]. To make OCT more applicable to the clinical situation, we developed a handheld OCT unit that has proven highly effective in our preliminary clinical trials. OCT has proven especially valuable in studying the living kidney because not only are excisional biopsies invasive, damaging and can only sample a very small region of the kidney, they also produce severe artifacts that are difficult to distinguish from ischemia and other injuries [4]. In addition, OCT can detect blood flow in vivo using the Doppler [34]. Doppler OCT (DOCT) combines the ability of OCT to capture high-resolution structural images with corresponding Doppler velocity maps that can be merged together to identify regions with moving reflectors, indicating blood flow. Thus, OCT/DOCT is a powerful tool that combines structural and functional imaging which could be used to evaluate kidney status in vivo and in real-time during and following surgical procedures.

In this paper, we document the ability of OCT/DOCT to diagnose ATN in human donor kidneys both prior to and following their transplantation. We conclude that OCT imaging of donor kidneys can provide transplant surgeons with a means for predicting renal transplant outcomes.

\section{OCT System Setup and Design}

A custom-built OCT system with a fiber-optic, hand-held probe was used in this study, enabling real- time, intra-operative OCT imaging during kidney transplant procedures (Figures 1 and 2A,B). Briefly, the details of the OCT system used in our studies consisted of a Fourier-domain OCT system with swept-source laser operating at $1310 \mathrm{~nm}$ center wavelength and $100 \mathrm{~nm}$ bandwidth. The OCT system's axial resolution was $\sim 12 \mu \mathrm{m}$, and using a $2.0 \mathrm{x}$ objective in the sample arm, a transverse resolution of $\sim 15 \mu \mathrm{m}$ was achieved as determined by resolution chart. Wavelength sweeping frequency was $16 \mathrm{kHz}$ enabling real-time $2 \mathrm{D}$ imaging. OCT image pixel dimensions were as follows: $1024[\mathrm{X}]$ by $512[\mathrm{Z}]$, and the associated 2D image scan dimensions were $3.75 \mathrm{~mm}[\mathrm{X}]$ by $2.0 \mathrm{~mm}[\mathrm{Z}]$. The system's sensitivity was with $\sim 90 \mathrm{~dB}$ [31].

For operating room (OR) imaging, the hand-held OCT imaging device was assembled on a portable cart that can be easily wheeled into and out of the operating room. Figure $2 \mathrm{~A}$ shows the portable imaging system being used in the OR. The imaging system was equipped with two output monitors facing opposing directions. One monitor enabled the operator to visualize and record the data while the second monitor let the physicians visualize the imaging in real-time.

\section{Clinical Trials (Methods)}

Prior to engaging in this research, the protocol was approved by the Institutional Review Boards at both Georgetown University and the University of Maryland. Patients scheduled to receive kidney transplant at Georgetown University Medical Center (Georgetown, Washington D.C.) were enrolled in this study. Informed consent was obtained from all patients prior to imaging. A total of 28 patients were enrolled in this study and for each patient we imaged the kidney prior to transplant (i.e., ex vivo) and following transplantation and reperfusion (i.e., in vivo).

Following procurement from the donor, the kidney was transferred to a sterile ice bath solution prior to its transplantation. During this time, the kidney was imaged using our hand-held OCT imaging probe (ex vivo kidney). The entire hand-held probe with its six-foot length of cords was covered with a sterile camera sleeve. A $1.5 \mathrm{~cm}$ circular hole was cut in the end of the sleeve and covered with a commercially available, sterile, transparent "Tegaderm" film (3M Health Care, St. Paul MN). This set-up provided a sterile and moisture barrier without impeding the imaging laser beam. It took approximately 2-4 minutes to image the entire kidney ex vivo. Following transplantation of the kidney into the patient and reestablishment of blood flow to the donor kidney, we imaged the transplanted kidney again (in vivo kidney). For in vivo kidney imaging, the interference fringe data (the complex OCT signal including both magnitude and phase information) was recorded to enable DOCT processing and analysis. The total time for surveying the transplanted kidney was approximately 2-4 minutes.

\section{Clinical Trials (Results)}

\section{Ex vivo Kidney Imaging}

While in a sterile ice bath prior to transplantation into the recipient, the entire donor kidney surface was surveyed by OCT (i.e., surveyed globally). Figure 3 shows representative OCT imaging of the ex vivo kidneys prior to transplantation. The kidney parenchyma containing uriniferous tubules is visible beneath the intact kidney capsule. There were significant variations in the openness of lumens of the

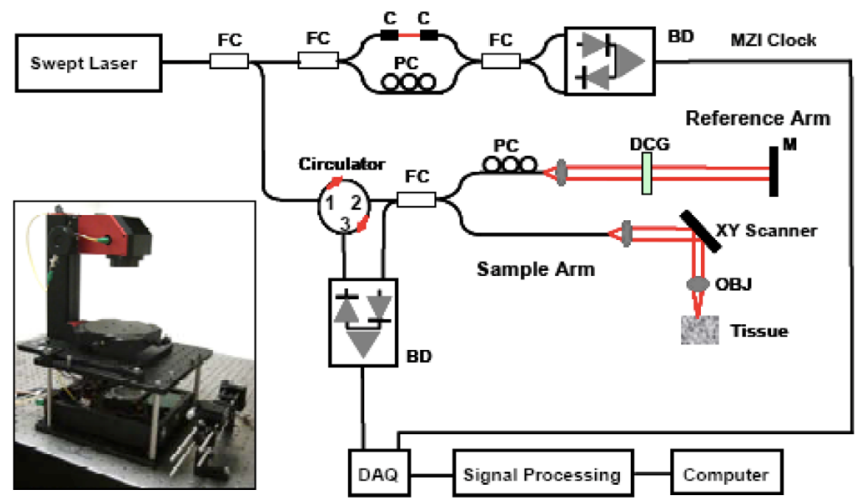

Figure 1: Schematics of the Fourier-domain OCT system used in kidney imaging studies. Inset shows the photo of OCT microscope.
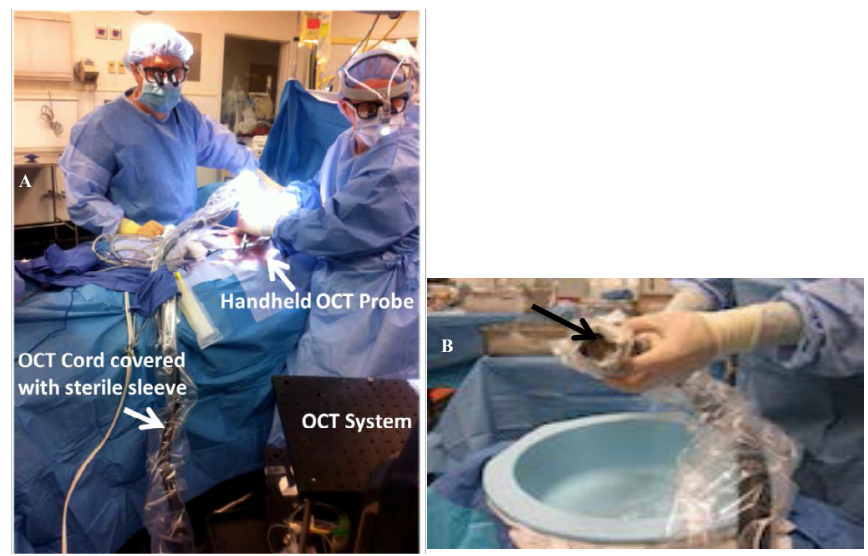

Figure 2: A. Transplant surgeons using the sterilized hand-held OCT probe to image a transplanted human donor kidney in the OR. Both surgeons are looking at real-time images of the functioning kidney while they are imaging it. The OCT probe and associated wires are covered with a sterile camera sleeve. B. The OCT imaging probe covered with camera sleeve and transparent Tegaderm (arrow). 
Citation: Andrews PM, Chen Y (2014) Using Optical Coherence Tomography (OCT) to Evaluate Human Donor Kidneys Prior to and Following Transplantation. J Nephrol Ther 4: 151. doi:10.4172/2161-0959.1000151

proximal convoluted tubules (Figures $3 \mathrm{~A}$ and $\mathrm{B}$ ). An analysis of posttransplant function (i.e., serum creatinine and BUN values) revealed that a decrease in the proximal convoluted tubule luminal diameters correlated closely with a poorer post-transplant renal function.

\section{In vivo OCT Kidney Imaging}

Following transplanting and reestablishing blood flow to the donor kidney, the kidney was imaged using OCT (Figures 4 and 5) and DOCT (Figure 6). Figure 4 depicts representative in vivo kidney OCT images from two patients (Figure $4 \mathrm{~A}$ and $3 \mathrm{~B}$ are from one, $4 \mathrm{C}$ and $4 \mathrm{D}$ are from another) showing cross-sectional profiles of superficial proximal tubules below the renal capsule. The openness of tubule lumens reflects a functioning post- transplanted kidney. The poorest post-transplant function was seen in one patient who had suffered an additional normothermic ischemic insult during reimplantation. This patient's transplanted kidney did not show open tubules and suffered DGF.

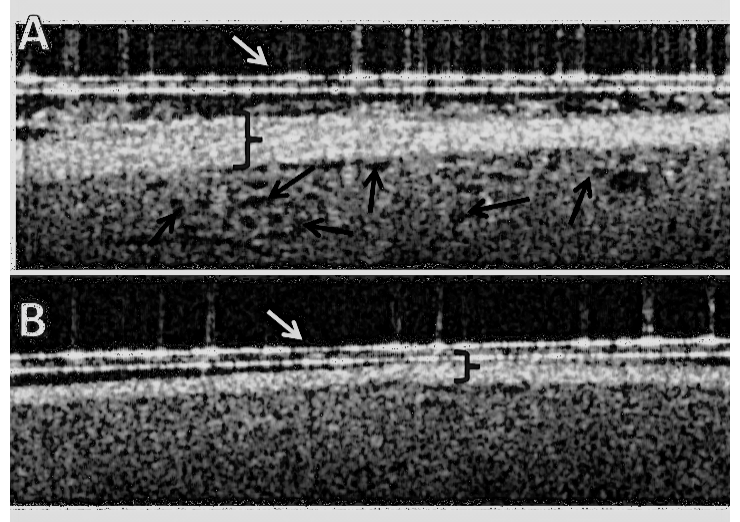

Figure 3: OCT image of a donor kidney showing open tubules (black arrows) prior to transplantation (A) and a donor kidney showing no open tubules prior to transplantation (B). The donor kidney seen at $A$ exhibited a rapid return to normal function (two days), while that at "B" did not recover normal values until nearly two weeks. The brackets indicate the kidney capsule while the white arrows indicate the Tegaderm. Scale bar $=500 \mu \mathrm{m}$.
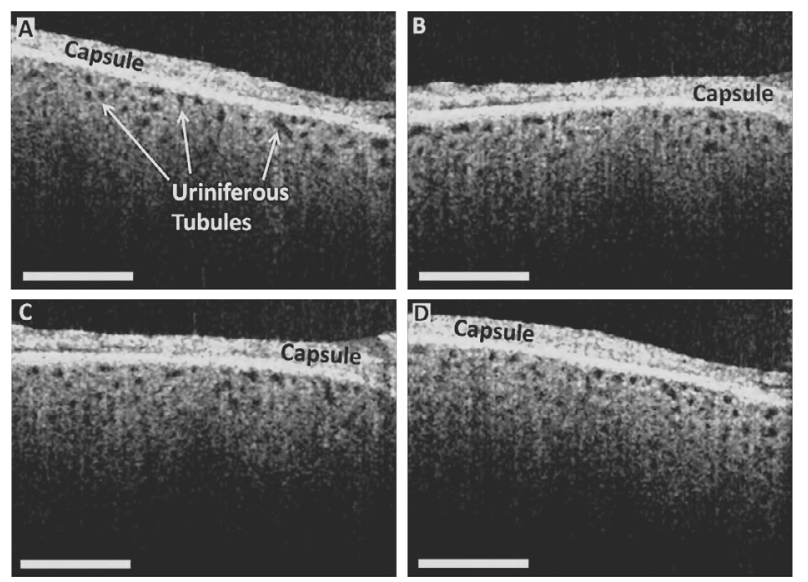

Figure 4: OCT imaging of human kidneys following transplantation showing open uriniferous tubules below the renal capsule in two different patients ( $A$ and $B$ are for one patient and $C$ and $D$ are for another). Tubules appear to be open with some degree of homogeneity throughout the images. Scale bar = $500 \mu \mathrm{m}$. (Reprinted with permission from JIOHS, 7 (4), 130064, 2014).
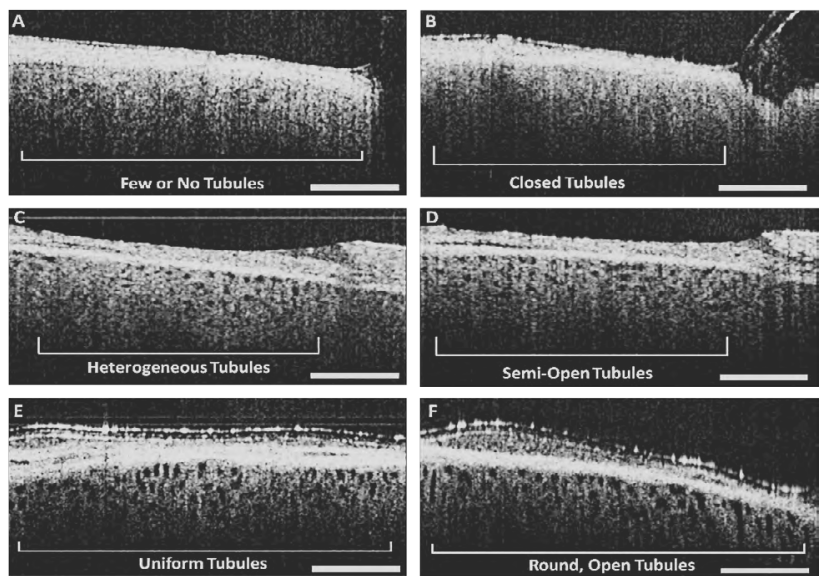

Figure 5: Examples of variation of in vivo OCT images of human kidneys for tubule size/shape and density/uniformity. Tubule size/shape: (B) poor (D) moderate (F) good. Tubule density/uniformity: (A) poor (C) moderate (E) good. Scale bar=500 $\mu \mathrm{m}$. (Reprinted with permission from JIOHS, 7 (4), 130064, 2014)
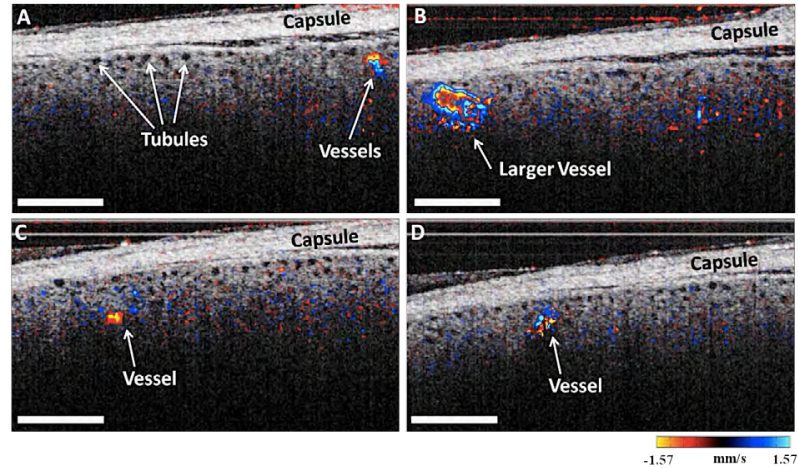

Figure 6: In vivo human kidney showing open tubules and cortical blood flow Open tubules appear round and relatively uniform. A larger blood vessel is seen in (B) against some smaller vessels observed in (A, C, and D). The smaller blue and red dots represent peritubular vessels. Data are from a single patient. Scale bar $=500 \mu \mathrm{m}$. ((Reprinted with permission from JIOHS, 7 (4), 130064, 2014).

The spatial distribution of opening tubule lumens exhibited variation from patient to patient. Example images of the variation of tubule morphology for both the size/shape and the density/uniformity are evident in Figure 5. Tubule size/shape was grouped to poor, moderate, and good. Tubule density/uniformity was also grouped to poor, moderate, and good categories. These images illustrate the visual appearance represented by the scoring values and can be used for standardizing the scoring system across multiple users for further analysis of tubule opening quantification.

OCT fringe data was also recorded during in vivo imaging to enable DOCT imaging for visualizing blood flow in real time as shown in Figure 6. OCT is displayed in gray scale and DOCT is overlaid with a color map. Blue-cyan represents blood flow in one direction while red-yellow represent blood flow in the opposite direction. In a previous publication, we have shown that DOCT will reveal glomerular blood flow and can be used to evaluate glomerular function/status [31]. 


\section{Future studies}

The preliminary studies described in this paper demonstrate the feasibility of using OCT to assess donor kidney status prior to and immediately following transplantation by examining tubular morphology and renal blood flow. With $\sim 12 \mu \mathrm{m}$ axial and $\sim 15 \mu \mathrm{m}$ transverse resolution and $16 \mathrm{kHz}$ axial scan rate, OCT revealed renal tubules and cortical blood vessels in real-time during transplantation procedures. 3D imaging will be important in future OCT/DOCT kidney imaging as it provides not only comprehensive volumetric information, but also enables integration of DOCT signals over en face plane to quantify flow information correctly [32-34]. Due to significant motion from the hand-held scanner, 3D OCT imaging could not be performed in clinical settings with our current image acquisition speed. Higher speed imaging systems, however, are now commercially available. For example, Thorlabs Inc. recently developed a VCSELbased swept source laser running at $100 \mathrm{kHz}$ axial scan rate at 1.3 $\mu \mathrm{m}$ with $100 \mathrm{~nm}$ tuning range [35]. Alternatively, a Fourier-domain mode-locked (FDML) laser [36] enables ultrahigh-speed OCT imaging up to $\mathrm{MHz}$ A-scan rates $[37,38]$ and can be used for wide-field kidney imaging. These novel light sources will bring new clinical tools capable of volumetric imaging of tissue pathology.

Motion artifacts might be present during image acquisition especially during in vivo imaging. One can stabilize the kidney against the abdominal wall using standard instruments to reduce motion artifacts while imaging. The OCT hand-held probe (using the "cage" as a spacer) can also be gently placed on the kidney surface to further minimize motion. Also, the OCT probe can be attached to an articulating arm to reduce the tremor of the surgeons' hand. Finally, cross-correlation algorithms can be applied to correct the motion artifacts [39].

\section{Conclusions}

Recent studies have shown that OCT/DOCT is a powerful emerging medical imaging technology that can be used to evaluate the human kidneys prior to and following their transplantation. Preliminary result demonstrate that OCT/DOCT is a safe, non-invasive procedure that can assess donor kidney status in a timely fashion in the OR and provide important information that can be used to predict posttransplant kidney function.

\section{Acknowledgements}

We acknowledge the following individuals, all of whom contributed to the studies reviewed in this manuscript: Hsing-Wen Wang, Jeremiah Wierwille, We Gong, Jennifer Verbesey, Matthew Cooper, Seyed Ghasemian, Daniel Joh, Peter Alexandrov, Derek Rogalsky, Patrick Moody, and Allen Chen. We also thank Dr. James Jiang and Alex Cable from Thorlabs Inc. for technical support.

\section{References}

1. United States Renal Data System Annual Data Report (2009).

2. Perico N, Cattaneo D, Sayegh MH, Remuzzi G (2004) Delayed graft function in kidney transplantation. Lancet 364: 1814-1827.

3. Sanfilippo F, Vaughn WK, Spees EK, Lucas BA (1984) The detrimental effects of delayed graft function in cadaver donor renal transplantation. Transplantation 38: $643-648$

4. Andrews PM (1994) Noninvasive vital microscopy to monitor tubular necrosis of cold-stored kidneys. Transplantation 57: 1143-1148.

5. Andrews PM, Khirabadi BS, Bengs BC (2002) Using tandem scanning confocal microscopy to predict the status of donor kidneys. Nephron 91: 148-155.

6. Maunsbach AB (1966) The influence of different fixatives and fixation methods on the ultrastructure of rat kidney proximal tubule cells. I. Comparison of different perfusion fixation methods and of glutaraldehyde, formaldehyde and osmium tetroxide fixatives. J Ultrastruct Res 15: 242-82.

7. Campo-Ruiz V, Lauwers GY, Anderson RR, Delgado-Baeza E, González S (2006) Novel virtual biopsy of the kidney with near infrared, reflectance confocal microscopy: a pilot study in vivo and ex vivo. J Urol 175: 327-336.

8. Kang JJ, Toma I, Sipos A, McCulloch F, Peti-Peterdi J (2006) Quantitative imaging of basic functions in renal (patho)physiology. Am J Physiol Renal Physiol 291: F495-502

9. Dunn KW, Sandoval RM, Kelly KJ, Dagher PC, Tanner GA, et al. (2002) Functional studies of the kidney of living animals using multicolor two-photon microscopy. Am J Physiol Cell Physiol 283: C905-916.

10. Yu W, Sandoval RM, Molitoris BA (2007) Rapid determination of renal filtration function using an optical ratiometric imaging approach. Am J Physiol Renal Physiol 292: F1873-1880.

11. Huang D, Swanson EA, Lin CP, Schuman JS, Stinson WG, et al. (1991) Optical coherence tomography. Science 254: 1178-1181.

12. Fujimoto JG (2003) Optical coherence tomography for ultrahigh resolution in vivo imaging. Nat Biotechnol 21: 1361-1367

13. Hee MR, Izatt JA, Swanson EA, Huang D, Schuman JS, et al. (1995) Optical coherence tomography of the human retina. Arch Ophthalmol 113: 325-332.

14. CA Puliafito, MR Hee, JS Schuman, JG Fujimoto (1996) Optical coherence tomography of ocular diseases Thorofare, NJ: Slack Inc.

15. Wollstein G, Paunescu LA, Ko TH, Fujimoto JG, Kowalevicz A, et al (2005) Ultrahigh-resolution optical coherence tomography in glaucoma. Ophthalmology 112: 229-237.

16. Brezinski M (2002) Characterizing arterial plaque with optical coherence tomography. Curr Opin Cardiol 17: 648-655.

17. Jang IK, Bouma B, MacNeill B, Takano M, Shishkov M, et al. (2003) In-vivo coronary plaque characteristics in patients with various clinical presentations using Optical Coherence Tomography. Circulation 108: 373-373.

18. Bouma BE, Tearney GJ, Compton CC, Nishioka NS (2000) High-resolution imaging of the human esophagus and stomach in vivo using optical coherence tomography. Gastrointest Endosc 51: 467-474.

19. MV Sivak, K Kobayashi, JA Izatt, AM Rollins, R Ung-Runyawee, et al. (2000) High--resolution endoscopic imaging of the GI tract using optical coherence tomography. Gastrointestinal Endoscopy 51: 474-479.

20. Li XD, Boppart SA, Van Dam J, Mashimo H, Mutinga M, et al. (2000) Optical coherence tomography: advanced technology for the endoscopic imaging of Barrett's esophagus. Endoscopy 32: 921-930.

21. Y Chen, AD Aguirre, PL Hsiung, S Desai, PR Herz, et al. (2007) Ultra high resolution optical coherence tomography of Barrett's esophagus: preliminary descriptive clinical study correlating images with histology. Endoscopy 39: 599 -605 .

22. Welzel J, Lankenau E, Birngruber R, Engelhardt R (1997) Optical coherence tomography of the human skin. J Am Acad Dermatol 37: 958-963.

23. Otis LL, Everett MJ, Sathyam US, Colston BW Jr (2000) Optical coherence tomography: a new imaging technology for dentistry. J Am Dent Assoc 131 511-514.

24. D'Amico AV, Weinstein M, Li X, Richie JP, Fujimoto J (2000) Optical coherence tomography as a method for identifying benign and malignant microscopic structures in the prostate gland. Urology 55: 783-787.

25. C Pitris, A Goodman, SA Boppart, JJ Libus, JG Fujimoto, et al. (1999) High-resolution imaging of gynecologic neoplasms using optical coherence tomography. Obstetrics and Gynecology 93: 135-139.

26. GJ Tearney, SA Boppart, BE Bouma, ME Brezinski, NJ Weissman, et al. (1996) Scanning single-mode fiber optic catheter--endoscope for optical coherence tomography. Optics Letters 21: 543-545

27. Herz P, Chen Y, Aguirre A, Fujimoto J, Mashimo H, et al. (2004) Ultrahigh resolution optical biopsy with endoscopic optical coherence tomography. Opt Express 12: 3532-3542.

28. Li X, Chudoba C, Ko T, Pitris C, Fujimoto JG (2000) Imaging needle for optical coherence tomography. Opt Lett 25: 1520-1522.

29. Chen Y, Andrews PM, Aguirre AD, Schmitt JM, Fujimoto JG (2007) High- 
Citation: Andrews PM, Chen Y (2014) Using Optical Coherence Tomography (OCT) to Evaluate Human Donor Kidneys Prior to and Following Transplantation. J Nephrol Ther 4: 151. doi:10.4172/2161-0959.1000151

resolution three-dimensional optical coherence tomography imaging of kidney microanatomy ex vivo. J Biomed Opt 12: 034008.

30. Andrews PM, Chen Y, Onozato ML, Huang SW, Adler DC, et al. (2008) Highresolution optical coherence tomography imaging of the living kidney. Lab Invest 88: 441-449.

31. Wierwille J, Andrews PM, Onozato ML, Jiang J, Cable A, et al. (2011) In vivo, label-free, three-dimensional quantitative imaging of kidney microcirculation using Doppler optical coherence tomography. Lab Invest 91: 1596-1604.

32. Li Q, Onozato ML, Andrews PM, Chen CW, Paek A, et al. (2009) Automated quantification of microstructural dimensions of the human kidney using optical coherence tomography (OCT). Opt Express 17: 16000-16016.

33. Onozato ML, Andrews PM, Li Q, Jiang J, Cable A, et al. (2010) Optical coherence tomography of human kidney. J Urol 183: 2090-2094.

34. Srinivasan VJ, Sakadzic S, Gorczynska I, Ruvinskaya S, Wu W, et al. (2010) Quantitative cerebral blood flow with optical coherence tomography. Opt Express 18: 2477-2494.
35. Jayaraman V, Jiang J, Li H, Heim PJS, Cole GD, et al. OCT Imaging up to $760 \mathrm{kHz}$ Axial Scan Rate using Single-Mode 1310nm MEMS-Tunable VCSELs with $>100 \mathrm{~nm}$ Tuning Range, in Conference on Lasers and Electro-Optics: Applications and Technology 2011, Optical Society of America: Baltimore, MD. p. PDPB2.

36. Huber R, Wojtkowski M, Fujimoto JG (2006) Fourier Domain Mode Locking (FDML): A new laser operating regime and applications for optical coherence tomography. Opt Express 14: 3225-3237.

37. Tsai TH, Potsaid B, Kraus MF, Zhou C, Tao YK, et al. (2011) Piezoelectrictransducer-based miniature catheter for ultrahigh-speed endoscopic optical coherence tomography. Biomed Opt Express 2: 2438-2448.

38. Wieser W, Biedermann BR, Klein T, Eigenwillig CM, Huber R (2010) Multimegahertz OCT: High quality 3D imaging at 20 million A-scans and 4.5 GVoxels per second. Opt Express 18: 14685-14704.

39. Ahmad A, Adie SG, Chaney EJ, Sharma U, Boppart SA (2009) Cross correlation-based image acquisition technique for manually-scanned optical coherence tomography. Opt Express 17: 8125-8136. 